\title{
Paper
}

\section{Realtime System of Free Viewpoint Television}

\author{
(リアルタイム自由視点テレビシステム)
}

\author{
Purim Na Bangchang ${ }^{\dagger}, \quad$ Mehrdad Panahpour Tehrani ${ }^{\dagger \dagger}$, Toshiaki Fujii (member ${ }^{\dagger}$, \\ and Masayuki Tanimoto (member) ${ }^{\dagger}$
}

\begin{abstract}
In this paper, we propose a new 3D visual system, named "Free Viewpoint Television" or "FTV", which can acquire, process, and display any 3D scene in realtime. With this FTV system, users can freely control their own viewpoint and direction. The idea is based on the concept of ray-space, where 3D information can be represented by a group of light rays. A collection of light rays is obtained through an array of cameras, while the missing ray information between cameras is generated by interpolation. The first prototype based on this concept had both good image quality and a fast display rate.
\end{abstract}

Key words: FTV, Multi-Camera System, Free Viewpoint, Realtime System, Ray-Space Method.

\section{Introduction}

Among the different kinds of human communication conducted by mankind, visual communication, in which the information is carried in a visual form, is usually considered as the most illustrative, informative and comprehensive method. Although various kinds of visual materials has been utilized as basic communication elements, video-based visual communication has recently been considered as the most powerful and successful approach due to two main reasons. First, videobased communication can deliver larger amount of information than other image-based methods as temporal information is included. Second, it is not so complicated to perform the video-based communication. A video content can be produced, transmitted and displayed easily by inexpensive hardware.

The popularity of video-based method has been achieved through purely $2 \mathrm{D}$ imaging system and with viewers have no control over their own viewpoint. While most of us have adapted to these limitations, human visual systems are designed to see things in 3D manner and we normally expect to be able to change our view position around objects according to our interest. It is clear that this need can not be fulfilled with current

Reported in SPIE on Jan. 24, 2003

Received March 30, 2005; Revised April 26, 2005; Accepted June 13, 2005

† Department of Information Electronics, Graduate School of Engineering, Nagoya University

(Furo-cho, Chikusa-ku, Nagoya 464-8603, Japan)

$\dagger \dagger$ Information Technology Center, Nagoya University

(Furo-cho, Chikusa-ku, Nagoya 464-8601, Japan) video-based approach. Thus, the $3 \mathrm{D}$ visual communication, in which a $3 \mathrm{D}$ visual content is used as a communication component, has been highly desired for a long time. Similar to video-based system, the successfulness of $3 \mathrm{D}$ visual communication will depend on the ease in capturing, processing, and displaying $3 \mathrm{D}$ real-world scene information.

Based on this reason, the researches on $3 \mathrm{D}$ visual communication are emphasized mainly on a visual system that has abilities to perform those tasks, in realtime if possible. In the past few years, some $3 \mathrm{D}$ visual systems 1) 3) have been proposed. However, only few can operate in realtime. Because arbitrary view image generation is very expensive and complicated, it is very difficult to build a realtime system that operates continually at a constant speed. Some systems ${ }^{4) 5)}$ can achieve realtime operation by applying a simple interpolation. However, it can have some adverse effects on image quality especially in a complicated scene. Other approach is to reduce a reality level of output image ${ }^{6)}$ by mixing between real world information and pre-generated object models.

In this paper, we propose the new $3 \mathrm{D}$ visual system named "Free Viewpoint Television (FTV)". With this FTV system, users can experience $3 \mathrm{D}$ visual feeling as they can freely control their own view positions and directions as they desire. The system is based on concept of ray-space in which an arbitrary photo-realistic view can be generated from a collection of real view images. It aims for full realtime operation in which $3 \mathrm{D}$ visual information are acquired, processed and displayed 
all in realtime. The collection of images is obtained through an array of cameras where the missing ray information can be obtained by ray-space interpolation method. This approach leads to a simple, flexible and powerful system. For example, the FTV can be operated on any camera configuration without any major modification. Apart from that, the processing tasks of FTV can be shared to increase system's display frame rate easily.

The remaining of this paper is divided into 5 main parts. In section 2 , ray space method will be explained. In section 3 , the implementation of the FTV system will be covered in detail. In section 4 , performance of the first FTV prototype system will be reported. In section 5 , the conclusion of this paper will be given.

\section{Ray Space Representation}

In ray-space method $^{7) 8)}$, light ray is proposed as a basic element to representing visual information. Thus, $3 \mathrm{D}$ visual content of interested scene can be represented by a group of rays passing through scene's boundary. The group of rays is called "ray-space data".

Fig. 1 shows an example of the definition of rayspace. Let $(x, y, z)$ be three space coordinates, and $(\theta, \phi)$ be the parameters of direction. A ray going through free space can be uniquely represented by intensity function in this $5 \mathrm{D}$ space as $f(x, y, z, \theta, \phi)$. The basic idea of ray-space is, in fact, this is a $5 \mathrm{D}$ function can be expressed by only $4 \mathrm{D}$ subspace of $f(x, y, \theta, \phi)$, intersects with the plane $z=0$. Thus, all the intensity data of rays can be expressed by equation (1). This ray parameter is called ray-space.

$$
f(x, y, \theta, \phi),-\pi \leqq \theta<\pi \text { and }-\frac{\pi}{2} \leqq \phi<\frac{\pi}{2}(1)
$$

Now, the relationship between the rays that pass through a point and its locus in the ray-space will be examined. For simplicity, we consider only a $2 \mathrm{D}$ rayspace $f(x, \theta)$ in which the vertical parallax $(\phi)$ and vertical position $(y)$ are neglected. Let $X, Z$ be real-space coordinates, and $x, u$ be the ray-space coordinates. The rays that pass through a point $P(X, Z)$ in the real-space form a line in the ray-space, given by

$$
X=x+u Z, u=\tan \theta
$$

It gives the most important and interesting characteristic of the ray-space representation that a view image corresponds to a cross section image of the ray-space data. Therefore, the view acquisition/display process can be considered the recording/extracting process of

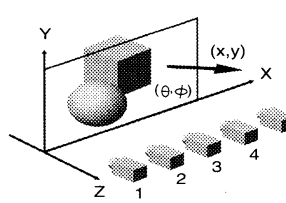

(a)

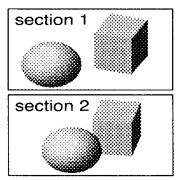

(d)

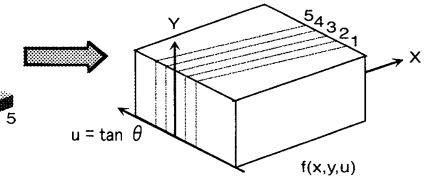

(b)

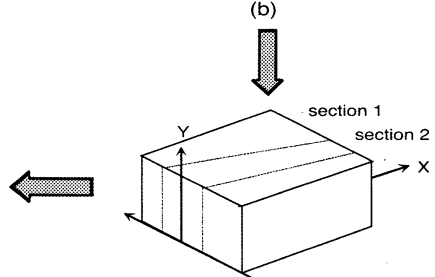

(c)
Fig. 1 Ray-space method (a) Ray space recording (b) Recorded ray-space (c) New view generation (d) Generated views.

the ray-space data along the locus. In the acquisition process, the ray data in the real space can be sampled and recorded as the ray-space data along the locus as shown in Fig. 1(b). Then, in the display process, the ray space data are cut along the locus and extract the section image of the ray space as shown in Fig. 1(c) and Fig. 1(d).

\section{Proposed FTV System}

Here, in the main section of the paper, we discuss on design and implementation of $\mathrm{FTV}^{9}$ system taken places from both theoretical and practical points of view. Nevertheless, the main emphasis is given to the practical side and how the first FTV prototype system is constructed. Although view image generation of FTV system is based on the ray-space representation concept explained in the previous section, the FTV system is designed to real time operation, in which images should be generated continuously at a constant frame rate. Thus, the designs of both system hardware and algorithms emphasize to optimize the balance between image quality and processing speed.

This section is further separated into two subsections, system hardware and system algorithm.

\section{1 System Hardware}

The discussions of FTV hardware are divided into two main topics, cameras system for capturing dynamic ray-space data and computer network system for image generation processing sharing.

Dynamic ray-space data are simultaneously captured through a group of CCD cameras which can be arranged in either line or surface. Random arrangement of cameras is possible. However, as same as in ray-space representation theory, camera positions must be able to be expressed in a mathematically to reduce the parame- 


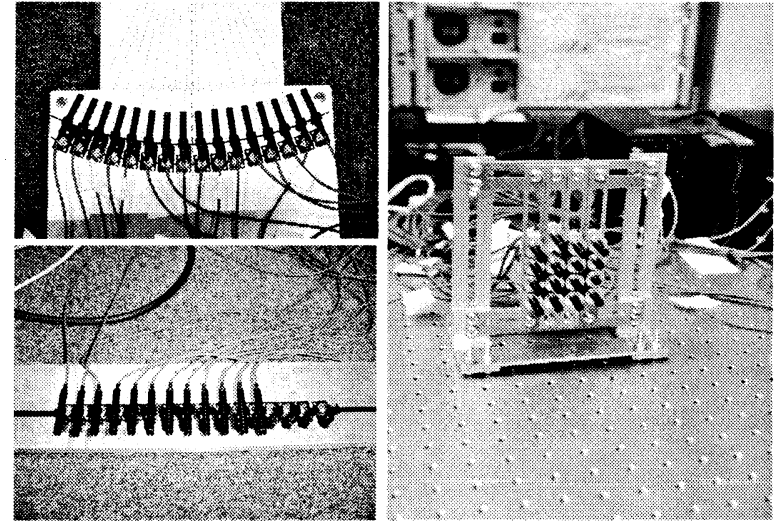

Fig. 2 Camera configuration. (top left) 1D line camera array, (bottom left) $1 \mathrm{D}$ arc camera array, (right) $2 \mathrm{D}$ camera array.

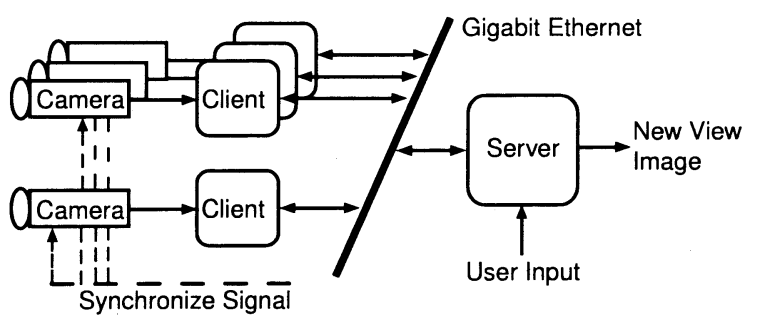

Fig. 3 FTV basic hardware configuration.

ters in the algorithm. For this prototype system, 16 CCD cameras are used. They can be arranged in three configurations, 1D line, 1D arc and 2D camera arrays. The pictures of the three camera configuration can be demonstrated as in Fig. 2. Those camera platforms are specially designed so distance between cameras can be adjusted freely.

FTV computer network system is constructed by two computer types called server and client computer. Main responsibility of server machine is user interface while main responsibility of client computer is camera control. Thus, server computers determine the number of users while client computers determine the number of view cameras. In this prototype system, 16 client nodes plus 1 server node are installed. All the computers are connected together forming a network through Ethernet connection. In order to keep all cameras synchronize, a synchronized signal is sent to all camera nodes at 30 pulses/sec. The basic hardware configuration can be depicted in Fig. 3. Details of each hardware component are given in table 1. All the components are based on only consumer-class hardware without any additional special hardware such as special camera or depth sensor.

\subsection{System Algorithms}

In this section, full discussion on system algorithms is given. First, the basic concept and overall algorithm
Table 1 Hardware specification

\begin{tabular}{l|l}
\hline \hline Component & Description \\
\hline Server Computer & CPU: Pentium III 850MHz Memory: 256MB \\
\hline Client Computer & CPU: Pentium III 800MHz Memory: 128MB \\
\hline Network & Star Network with 100MB Ethernet \\
\hline
\end{tabular}

will be covered. Then, more detail of each important part will be further discussed.

- Overall System Algorithm

Based on processing sharing, the proposed FTV system can be operated in 2 modes, centralized processing and decentralized processing. In the first approach, all interpolation tasks, which is the FTV's main processing task, are performed only in the server machine without any sharing. While, in second approach, the interpolation tasks are shared with all the client computers. The theoretical point of view of these two sharing approaches has already been fully discussed ${ }^{10)}$. In general, centralized processing is suitable when image transmission delay is high comparing with total processing time while decentralized processing is suitable when image transmission delay is low comparing with total processing time.

The basic flowcharts of centralized and decentralized processing approach are shown as in Fig. $\mathbf{4}$ and Fig. $\mathbf{5}$ respectively. The left side shows server processing task while the right side shows client processing task. The arrows shown in the diagram represent data transmission between clients and server where direction of arrow's head represents the direction of transmission.

For centralized processing, server is responsible for all important tasks including user interface, client controlling and view interpolation, while the clients are responsible for only image capturing. In order to generate a new view, server sends a "start signal" to all clients. The use of the start signal is to enforce all client processor to capture images simultaneously. When clients receive the start signal, view images are captured. Each captured image contains some offset that need to be corrected by image rectification. Then, all captured images are sent to server. After receiving the images, server performs interpolation. For 1D camera array, only horizontal interpolation is performed while, for $2 \mathrm{D}$ cases, both horizontal and vertical interpolations are performed. Then, based on interpolation results, server can generate and display the requested arbitrary view image. For each new view image generated, server needs to wait until it is time for the next frame image to be process, and the whole process restarts again.

In decentralized processing, the capturing of view im-

(65) 1193 


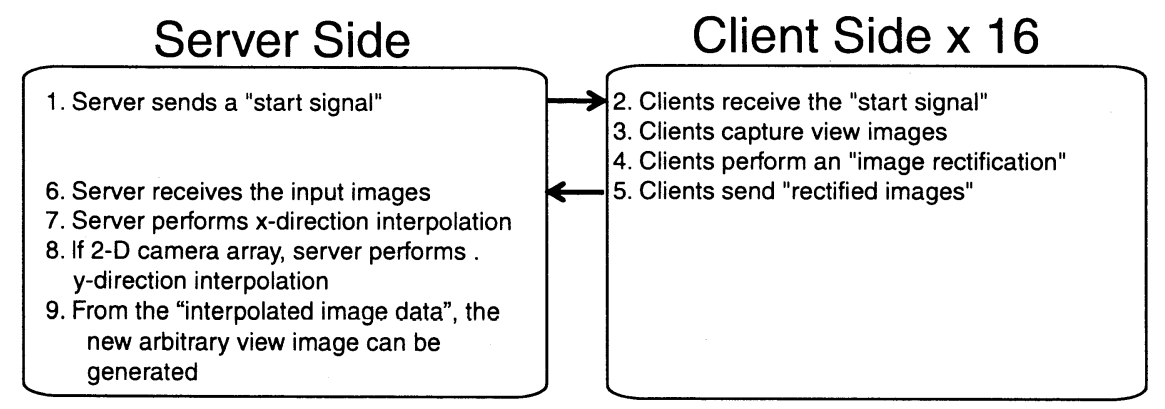

Fig. 4 Basic system flowchart for centralized approach.

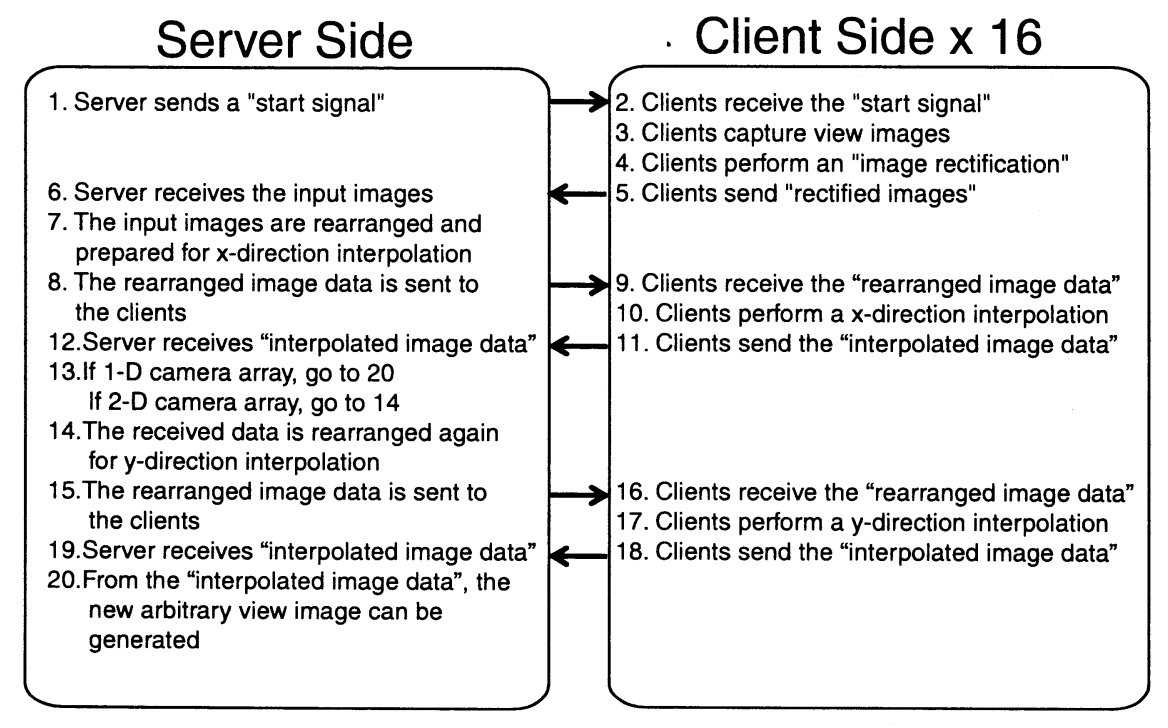

Fig. 5 Basic system flowchart for decentralized approach.

ages is similar with centralized one but interpolation tasks are performed in client computer. After receiving the input images, server rearranges the data for interpolation in horizontal direction. The new rearranged data is then sent back to the clients where the horizontal direction interpolation will be further performed. Next, the horizontal interpolated data is then sent back to the server again. For one-dimensional camera array, the interpolated data at this stage can be combined to generate the final new view image. For two-dimensional camera array, the process needs to be repeated for interpolation in vertical direction before the final new view image can be generated.

\section{- Rectification}

Generally when the multiview images are captured from different cameras, the accurate and arbitrary view generation requires that the images are rectified. Rectification determines a transformation of each image plane such that pairs of conjugate epipolar lines become collinear to one of the image axes. The important advantage of rectification is that in computing correspondences, a 2-D search problem is reduced to 1-D search problem. However, the common rectification methods are limited for only stereo-pair camera system. Therefore, according to the FTV camera configuration, we need to develop a new simplified rectification algorithm, which can be used for multi-view images.

Our rectification is performed by mapping all the input images into new rectified plane. The position of new plane should be the position of the interested objects. - The mapping process is based on the simple projective image mapping which mapping matrixes are calculated by using a calibration pattern. At the new positions, all the corresponded pixels in the horizontal direction should lie on the corresponding vertical position, and all the corresponded pixels in the vertical direction should lie on the corresponding horizontal position. By using this technique, we can ensure that the effects of camera dissimilarities at interested zone will be minimized.

\section{- Interpolation}

FTV system requests that dense ray-space data must be captured. However, it is very difficult and expensive task due to hardware limitation. Thus, in practical, only partial ray data of interested scene are acquired. 


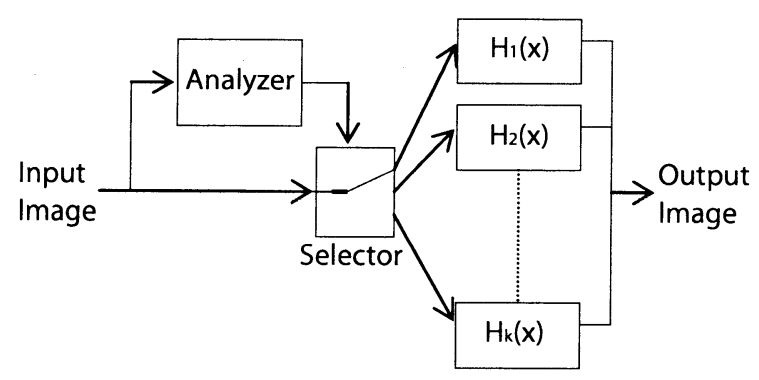

Fig. 6 Basic diagram of adaptive filter interpolation.

Then, the missing ray information between cameras is generated by interpolation. Thus, the interpolation method can have large effects on system's characteristics, especially frame rate and output quality, and can be considered as the most important part of the FTV system.

The interpolation technique proposed with this FTV system is based on the technique call "adaptive filtering ray-space data interpolation ${ }^{11) \sim 13)}$, which intended to replace a simple linear interpolation technique. It is designed based on the special characteristics of ray-space data in which slope of line in ray-space corresponds to depth of point in real-space.

The basic idea of this technique is a pre-constructed set of filters is used to determine a locus of real-world point in ray-space as shown in Fig. 6. First, a set of filters are prepared. The number, size and shape of filters are determined by interpolation parameters such as number of interpolated viewpoints, maximum disparity and searching step. An example of searching filters can be shown as in Fig. 7. Next, the input images are converted into ray-space data or EPI image. Each ray-space data is, then, up-sampled. The numbers of up-sampling lines are calculated by the number of interpolated views. After that, we can apple the filter set to test the input ray-space data. For each requested image pixel, the system try to choose the optimum filter based on block-based Mean Square Error (MSE) testing. The filter that gives the lowest MSE is selected for interpolating the requested pixel as shown in Fig. 8. Although the optimum filter is selected based on the block searching, the interpolated pixel value is calculated using only the center pixel values of the blocks.

\section{Interpolation for Dynamic Ray-Space Data}

The interpolation explained in the previous section is originally designed for interpolating static ray-space data in which temporal information is not valid. However, in the case of dynamic ray-space data as in FTV system, this technique can be modified to suit with new

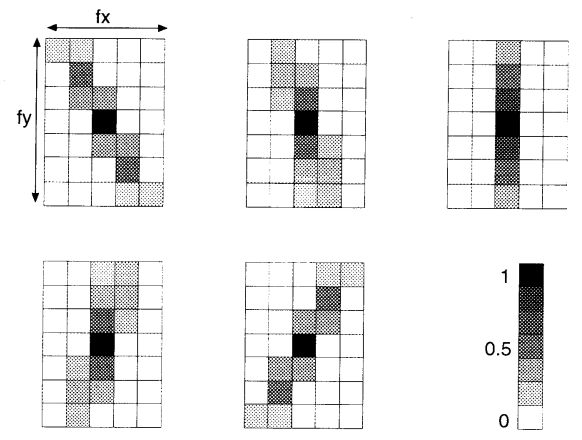

Fig. 7 Example of a set of filters.

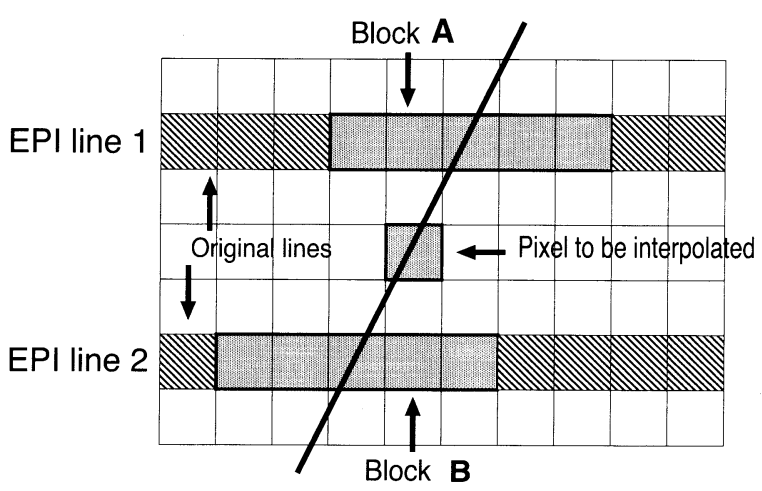

Fig. 8 Block matching and calculation of requested pixel.

characteristics. The modified version of this technique has been proposed ${ }^{14)}$. It is based on the idea that temporal information can be used to speed up interpolation process.

Sequences of input images are separated into a group called "group of picture" (GOP). The first image of each GOP is used as a reference frame and it is interpolated by the common interpolation method. The result of this interpolation is kept for further use. Other images in the GOP are interpolated based on the interpolation result of reference frame and motion information from reference frame to the current frame. The images of current frame are compared with images of reference frames in order to distinguish moving objects from background. In background part, filter searching is not needed as the filter value can be taken from the interpolation result of reference frame. While in moving objects part, the interpolation can be performed as usual. By using this technique, the interpolation speed can be increased dramatically with very small degradation of image quality.

\section{Interpolation for 2D Camera Configuration}

It is very simple to extend the concept from $1 \mathrm{D}$ camera array to $2 \mathrm{D}$ camera array as the computation can be performed one direction at the time. Thus, interpolation of $2 \mathrm{D}$ camera system is merely two passes of $1 \mathrm{D}$ camera interpolation. In the first step, the system per- 


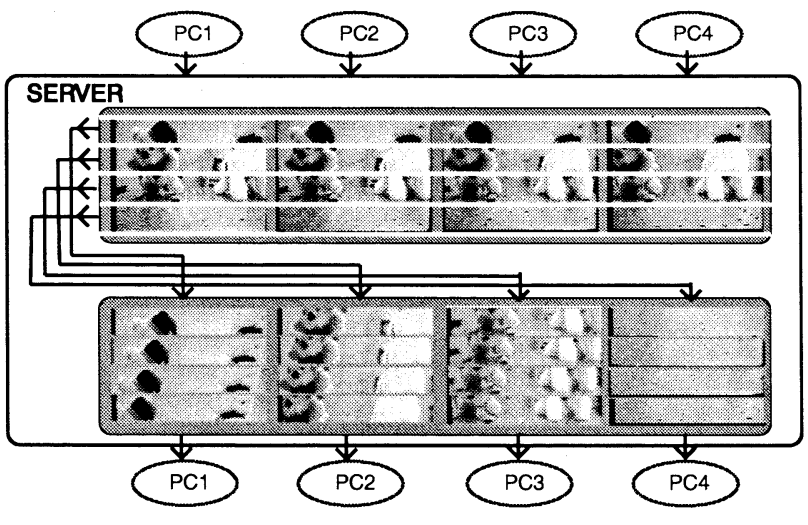

Fig. 9 Example of data arrangement for decentralized processing in the case of 4 sharing nodes.

forms calculations based on horizontal direction. Then the calculated results are passed to the second step. In the second step, the system performs calculations again based on vertical direction. Then the final output can be constructed.

\section{- Processing Sharing}

This processing sharing is the main algorithm that applied only to decentralized processing approach. It is based on the idea that system's frame rate can be increased by sharing processing tasks with all computers in FTV system. Each client computer individually processes only a small section of the whole image data. In order to perform sharing, each input image needs to be rearranged into some suitable form for sharing. This rearrangement is based on two factors, camera configuration and number of sharing nodes. Camera configuration determines how images are partitioned while the number of nodes determines the number of partitioning sections. For example, Fig. 9 shows how data are rearranged when the interpolation is in horizontal direction using four shared computers. In this case, each input image is partitioned into 4 sections along the horizontal axis. The first sections of each image are grouped together and sent to computer1 (PC1). Then, the process repeats for the next section for all remaining computers.

\section{Experimental Results}

In this section, the performance of the actual prototype system proposed in previous section will be evaluated based on two main aspects, display frame rate and image quality.

\subsection{Experimental Configurations}

FTV is a big system that contains many important parameters with various configurations. So, in order to simplify the experiments, only two main aspects were considered:
Table 2 Summary of important experimental parameters.

\begin{tabular}{|c|c|c|}
\hline Camera & Parameter detail & Experimental value \\
\hline \multirow{5}{*}{ 1D Line } & - Distance between cameras & $2 \mathrm{~cm}$ \\
\hline & - Distance from object plane & $30 \mathrm{~cm}$ \\
\hline & - Number interpolated images & 15 views \\
\hline & between cameras & \\
\hline & - Total available view position & 241 views \\
\hline \multirow{5}{*}{ 1D Arc } & - Distance between cameras & 3 degree \\
\hline & - Distance from object plane & $35 \mathrm{~cm}$ \\
\hline & - Number interpolated images & 15 views \\
\hline & between cameras & \\
\hline & - Total available view position & 241 views \\
\hline \multirow{5}{*}{$2 \mathrm{D}$} & - Distance between cameras & $2 \mathrm{~cm}$ \\
\hline & - Distance from object plane & $30 \mathrm{~cm}$ \\
\hline & - Number interpolated images & 7 views \\
\hline & between cameras & \\
\hline & - Total available view position & 625 views \\
\hline
\end{tabular}

1) Camera configuration. It can be separated into three groups as mentioned in section 3.1, 1D line, 1D arc and 2D camera array. The details of each camera configuration can be concluded as in Table 2 .

2) Processing algorithm approach. It can be separated into two main approaches as mentioned in section 3.2 , centralized processing and decentralized processing. Each processing approach is tested with both static data interpolation and dynamic data interpolation.

Note that the prototype system was first tested with various image sizes. From that test, we realized that the most suitable image size for this system is $160 \times 120$ pixel resolution. Thus, the experiments are performed based on this image resolution.

\section{2 System Processing Speed}

Fig. 10 and 11 show the prototype FTV system's display frame rate of various combinations between camera configuration and algorithm approach. Fig. 10 shows the result of static ray-space interpolation while Fig. 11 shows the result of dynamic ray-space interpolation. It is clear that, for this prototype system, the decentralize processing outperforms the centralized processing approach about three times. By applying the dynamic ray-space data interpolation, the system's frame rate can be further increase.

The 1D line camera array can obtain the highest frame rate due to its simplicities of representing rayspace data. While, the $2 \mathrm{D}$ camera array obtains the lowest frame rate due to it has to perform two-pass interpolations to generate one view image.

\section{3 Image Quality}

In order to measure image quality, the PSNR measurement is used in general. However, in this case, the PSNR does not work because reference images are not available. Thus, discussion of image quality has to rely 


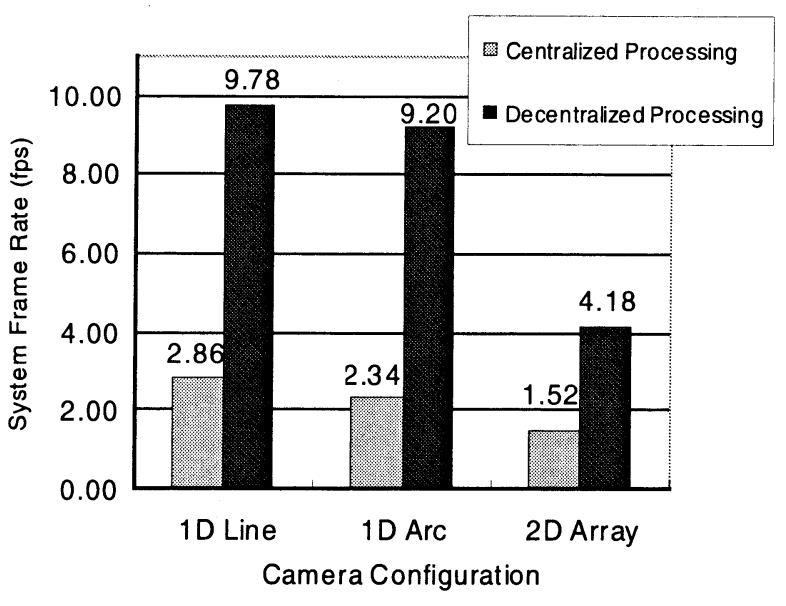

Fig. 10 Frame rate of various camera configurations and processing method (Static Interpolation).

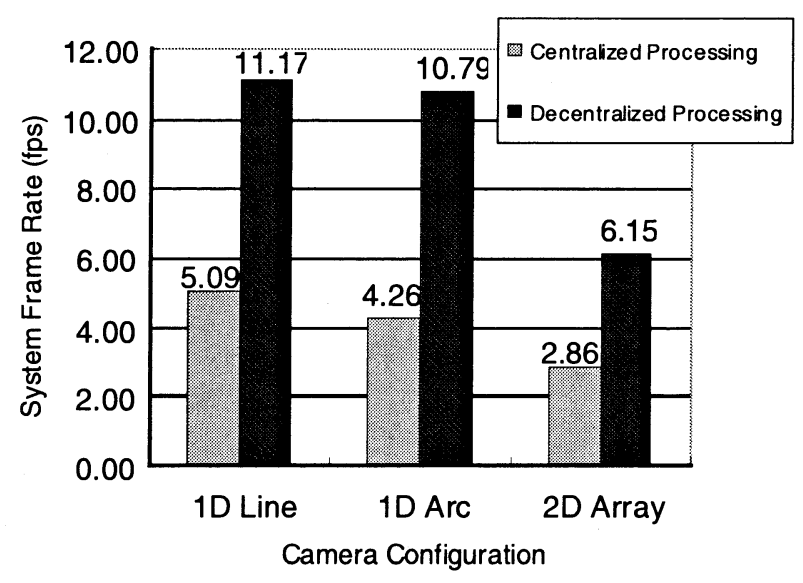

Fig. 11 Frame rate of various camera configurations and processing method (Dynamic Interpolation).

on only viewer's decision.

Figure 12 shows the array of rectified input images captured by 2-D camera array configuration while Figure 13 shows the rendering image result. Figure 13(a) shows the rendering images from different viewpoints on the camera plane while Figure 13(b) shows the gener-

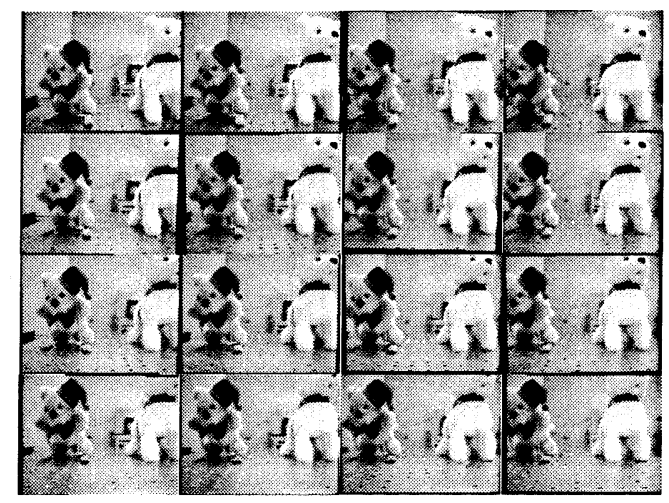

Fig. 12 Array of rectified input images. ated views as the viewpoint position moved away from the camera plane. When virtual camera moves away from the camera plane, the rays to generate the full image is inadequate so only partial image was displayed. The three-dimensional visual effect, such as occlusion effect caused by the dog dolls, can be demonstrated clearly when visual camera moves back and forth from the camera plane. The moving human hand, one of the difficult shapes to make a model or to interpolate correctly, is used to represent the dynamic object. Our system shows that it can handle the fast moving hand without any difficulty.

\section{Conclusions and Future Works}

In this paper, we introduced the experimental system of Free Viewpoint Television, a new realtime 3D visual communication system, and how the actual system was constructed. We also demonstrated that the proposed system has the ability to generate the arbitrary view image of various dynamic scenes or objects in realtime without any need of object model or pre-acquired depth information. Overall performances such as constructed image quality and display frame rate under this prototype system are impressive.

Even the output image size was small when comparing with nowadays standard, for real applications, the output images can be easily enlarged by simple image magnification without significant loss in display frame rate. As the result, the present prototype system of FTV can be applied to wide application areas including entertainments, educations, securities and environment monitoring.

Nevertheless, many aspects still need to be improved. Better image resolution and higher display frame rate are still preferred. Moreover, some mismatch errors that do occur in the output images need to be eliminated. In order to optimize the FTV usefulness, the captured data of dynamic scene must be able to store and transfer over existing hardware infrastructure. The main problem is, in general, dynamic ray-space data are very large. Thus, an efficient compression method should be researched intensively.

This work was supported by Japan Society for Promotion of Science (JSPS) under the Grant-in-Aid for Scientific Research (A) -15206046 -, and Ministry of Public Management, Home Affairs, Post and Telecommunication (MPHPT) under the grants on Strategic Information and Communication R\&D Promotion Scheme, and 

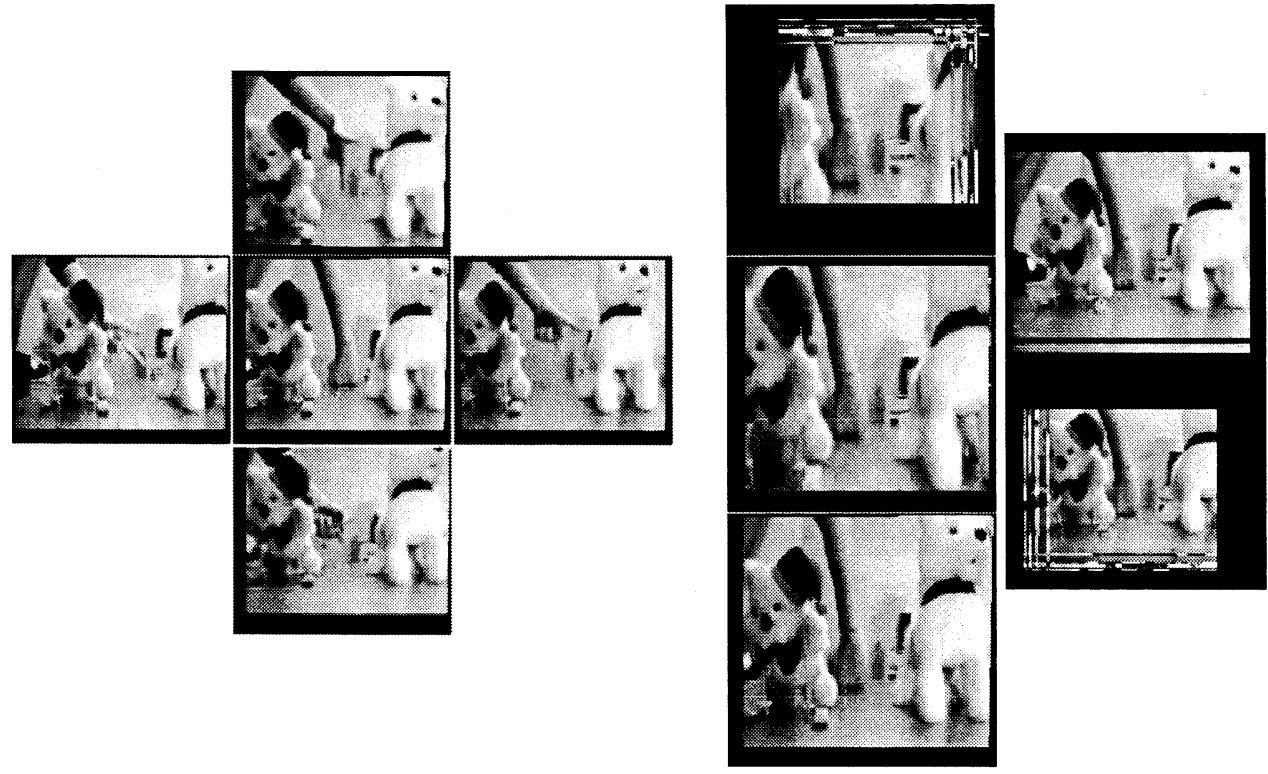

(a)

(b)

Fig. 13 Generated view image

(a) View images at various positions on camera plane

(b) Images at various positions away from camera plane.

Next-generation human interface -6196 .

\section{[References]}

1) H. Saito, S. Baba, M. Kimura, S Vedula, and T. Kanada, "Appearance-Based Virtual View Generation of TemporallyVarying Events from Multi-Camera Images in the 3D Room", Proceedings on 3-D Imaging and Modeling (Oct 1999)

2) D. J. Brady, R.A. Stack, S. Feller, E. Cull, L. Fernandez, D. Kammeyer, and R. Brady, "Information Flow in Streaming 3D Video", SPIE PRESS, CR76-13 (2000)

3) S. Wurmlin, E. Lamboray, O. G. Staadt, and M. H. Gross, "3D Video Recorder", Proceedings of Pacific Graphics'02, IEEE Computer Society Press, pp. 325-334 (2002)

4) T. Naemura, and H. Harashima,"Real-Time Video -Based Rendering for Augmented Spatial Communication", Visual Commun. Image Process, pp 620-631 (1998)

5) T. Naemura, J. Tago, and H. Harashima,"Real-Time Video Based Modeling and Rendering of 3D Scenes", IEEE Computer Graphics and App., pp 66-73, (2002)

6) T. Koyama, I. Kitahara, and Y. Ohta, "Live Mixed-Reality 3D Video in Soccer Stadium", Proceedings of IEEE on Mixed and Augment Reality (Oct 2003)

7) T. Fujii, T.Kimoto, and M. Tanimoto, "Ray space coding for $3 \mathrm{D}$ visual communication", Proceedings Picture Coding Symp.'96, 2, pp. 447-451 (March 1996)

8) T. Fujii, T. Kimoto, and M. Tanimoto, "A new flexible acquisition system of ray-space data for arbitrary objects", IEEE Trans. On Circuit and Systems for Video Technology, 10, 2, pp. 218-224 (March 2000)

9) P. Na Bangchang, T. Fujii, and M. Tanimoto, "Experimental system of free viewpoint television", Proceedings of SPIE, Stereoscopic Displays and Virtual Reality System, 5006-66, pp. 554-563 (Jan. 2003)

10) Mehrdad Panahpour Tehrani, Purim Na Bangchang, Toshiaki Fujii, Masayuki Tanimoto, "The Optimization of Distributed Processing for Arbitrary View Generation in Camera Sensor Networks", IEICE Transaction on Fundamentals of Electronics, Communication and Computer Sciences, E87-A, 8, pp. 18631870 (Aug. 2004)

11) Kobayashi , T. Fujii, T.Kimoto, and M. Tanimoto, "Interpolation of Ray-Space Data by Adaptive Filtering ", Proceedings of SPIE, Stereoscopic Displays and Virtual Reality System,San Jose, USA, 3958-34, (Jan 2001)

12) A. Nakanishi, T. Fujii, T. Kimoto, and M. Tanimoto, "Rayspace data interpolation for view image generation", Proceedings
IDW'01, pp. 1385-1388 (2001)

13) A. Nakanishi, T. Fujii, T. Kimoto, and M. Tanimoto, "Ray-space data interpolation by adaptive filtering using locus of corresponding points on epipolar plane image", The journal of the institute of Image information and Television Engineers (ITE), 56, 8, pp. 1321-1327 (Aug. 2002)

14) P. Na Bangchang, T. Fujii, M. Tanimoto, "Fast Ray-Space Interpolation for Multi-View Videos", Proceedings of the 10th International Display Workshops, IDW'03, Fukuoka, JAPAN, 3D3-3, pp. 1421-1424, (Dec. 2003)

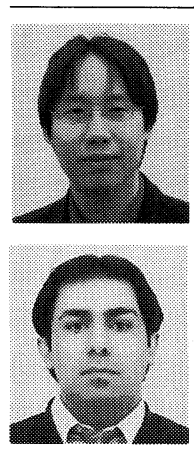

Purim Na Bangchang received the B.E. and M.E. degrees in Electronic Engineering from King Mongkut's Institute of Technology Ladkrabang, Thailand, 1997, and Nagoya University, Japan, 2004, respectively. His research interests include $3 \mathrm{D}$ visual communication, $3 \mathrm{D}$ system and $3 \mathrm{D}$ data compression.

Mehrdad Panahpour Tehrani received the B.E., M.E. and Dr.E degrees in Electrical Engineering from Tehran Polytechnics University, 1997, Tarbiat Modarres University-Tehran, Iran 2000, and Nagoya Unveristy, Japan, 2004, respectively. Currently, he is a Post-doctoral fellow at Information Technology Center, Nagoya University, Japan. His research interests include $3 \mathrm{D}$ image processing, coding, and $3 \mathrm{D}$ video/Audio intergration.

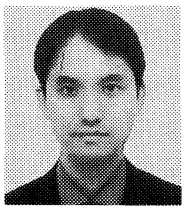

Toshiaki Fujii received the B.E., M.E., and Dr. Eng. degrees in electrical engineering from the University of Tokyo, Tokyo, Japan, in 1990, 1992, and 1995, respectively. He is currently an Associate Professor in the Graduate School of Engineering, Nagoya University, Japan. His research interests include $3 \mathrm{D}$ image processing and $3 \mathrm{D}$ visual communications.

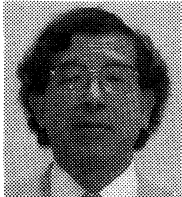

Masayuki Tanimoto received the B.E., M.E. and Dr.E. degrees in electronic engineering from the University of Tokyo, Tokyo, Japan, in 1970,1972 and 1976, respectively. Currently, he is a Professor of the Department of Information Electronics, Nagoya University, Japan. His current research interests include image coding, multidimensional signal processing, video processing, 3D and HD images, and multimedia systems. 